\title{
Fabrication of Aluminium-carbon fiber bridge, stump and pipe connector for Phase-II upgrade
}

\author{
Ngangkham Peter Singh1 \\ Indian Institute of Technology Madras \\ Chennai-36, India \\ E-mail: nag.pam@gmail. com
}

Rajesh Ravi Ranjan

Indian Institute of Technology Madras

Chennai-36, India

E-mail: 89rraviegmail.com

\section{S. Srinivasu}

Indian Institute of Technology Madras

Chennai-36, India

E-mail: devaduladittm.ac.in

\section{Prafulla Kumar Behera}

Indian Institute of Technology Madras

Chennai-36, India

E-mail: beheralitm.ac.in

\begin{abstract}
The CMS experiment will change its silicon tracker completely during phase-II upgrade. There is need to develop light and high precision and durable mechanical structure for silicon modules. The prime purpose of this should also be reducing material in the silicon tracker detector. The group at IIT Madras is involved in R\&D of production of this structures. The prime purpose of this should also be reducing material in the silicon tracker detector. We have produced high precision Al-CF bridges, stumps and Pipe connector 47 degrees for HgCAL detector.
\end{abstract}

The 27th International Workshop on Vertex Detectors - VERTEX2018 22-26 October 2018 MGM Beach Resorts, Muttukadu, Chennai, India

\footnotetext{
*Speaker.

$\dagger$ Acknowledgements: The authors of this paper would like to thank CERN for providing the design and the materials for this research and Finepart SwedenAB for assisting in micro-abrasive waterjet machining of these materials and above all Department of Sciences and Technology, Govt. Of India for providing fund for current research work. Communication:prafulla.behera@cern.ch
} 


\section{Introduction}

The Compact Muon Solenoid (CMS) experiment is going to be upgraded to phase-II for better sensitivity and resolution. Our group is involved in tracker sub detector. The pertinent component under consideration is strip-strip (2S) modules and its physics consequences can be found in technical design report of tracker for phase-II upgrade.

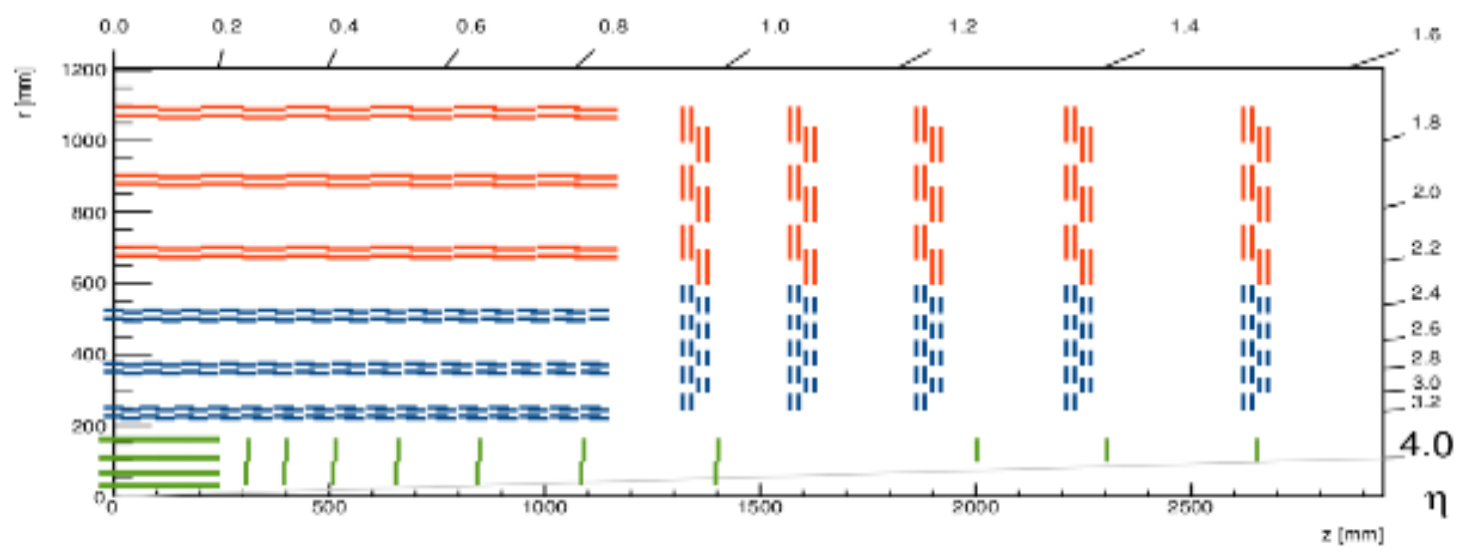

(a)

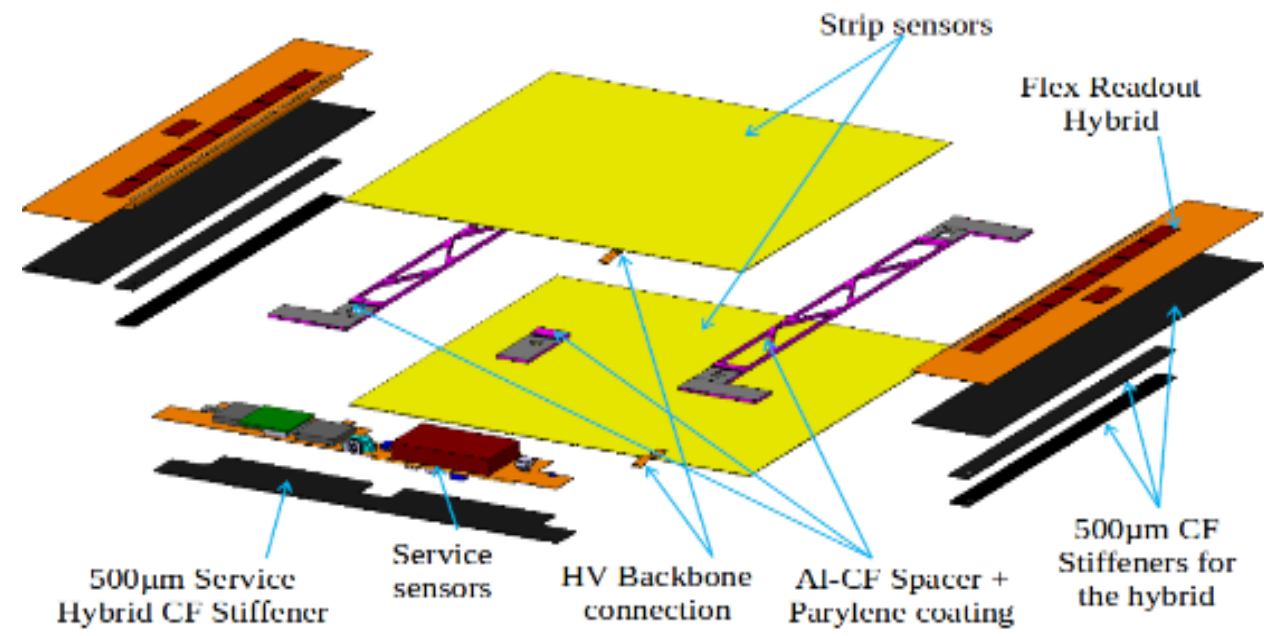

(b)

Figure 1: (a) Red bars are position of Strip-Strip (2S) modules within one quarter of outer tracker layout, (b) assembly of $2 \mathrm{~S}$ module.

The whole Si Tracker in LHC long shutdown 3 will be developed for detecting process for charged tracks as well as vertex related calculations. Few features of trackers are the new modules will have on-board P_t discrimination, decorrelation between two closely spaced sensor signal, improve local Pt measurement under strong magnetic field and rejects locally the low Pt tracks to minimize data volume. Level 1 (L1) and Readout will be at same time and Low power Gigabit 
Trans receiver (LP-GBT) as data link and DC-DC power convention will be integrated at module level. There are two primary modules has been proposed in this regard for optimization $2 \mathrm{~S}$ (StripStrip) module and PS (Pixel-Strip) module. The Carbon fiber reinforced Aluminium matrix composite or Aluminium-Carbon Fibre (Al-CF) and Carbon-fiber (CF) composite has been chosen as construction material for different geometrical parts of the whole structure. Al-CF composites has been used for bridge and stump while CF composite for fabricating different geometries of stiffeners. Macro abrasive waterjet (Macro- AWJ), micro abrasive waterjet (Micro-AWJ), milling processes has employed for fabricating these structures.

\subsection{Al-CF Bridge}

Aluminum carbon-fiber composites are advanced engineering composite materials developed for specific application like the stump and the bridge structure for 2S module (Fig. 1). Machining of such advanced composite material with conventional methods for achieving a complicated structure with $600 \mu \mathrm{m}$ wall thickness is difficult due to the fragility and softness of the material. Previous attempts for machining of Al-CF led to the formation of burrs with conventional milling and bending with slicing using wire-electric discharge machining [1]. Abrasive waterjets are considered to be one of the ideal method for machining advanced material due to its unique ability, such as minimal heat generation with negligible cutting force on almost any material [2-5].

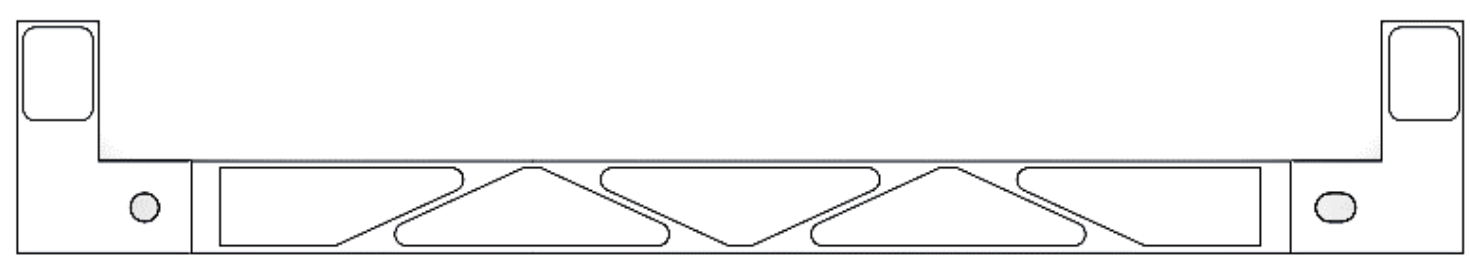

Fig. 2. Aluminum carbon fiber bridge structure

In this paper, two different approaches were identified for the fabrication of Al-CF bridge structure. In the first approach (Fig. 3), a multi-stage machining approach was used. Firstly, the Al-CF slices were cut using macro-abrasive waterjet machining. Secondly, the slices were flattened by polishing with emery sheets. Thirdly, the pockets and the profile along the boundary were generated by conventional milling on both sides using the specially designed fixture. Lastly, the complex structure in the bridge structure was generated using micro-abrasive waterjets. Special fixtures were designed and fabricated in-house for holding the workpiece for multiple stage. Fig. 4 shows the fabrication process of bridge fixture in multistages. 


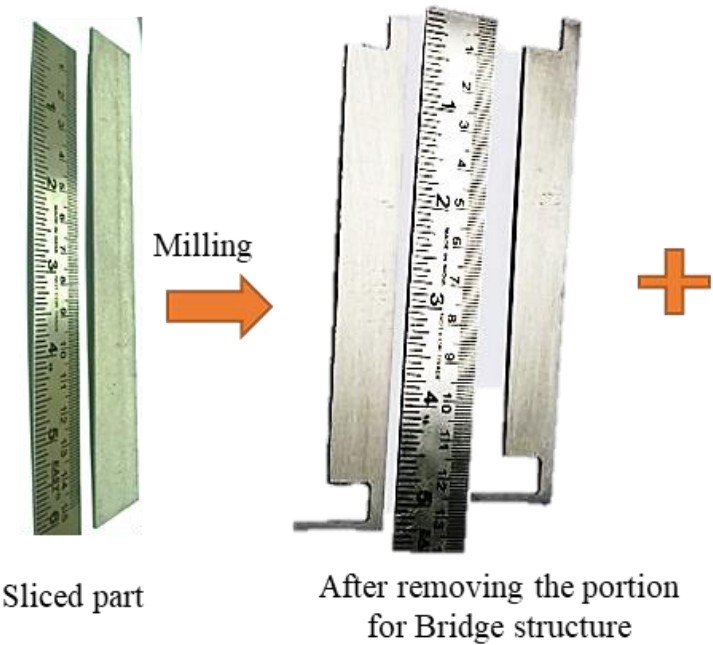

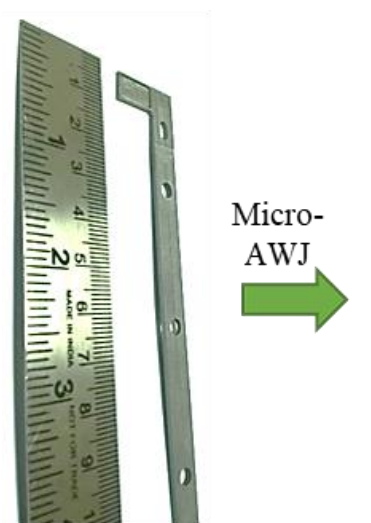

Milled part

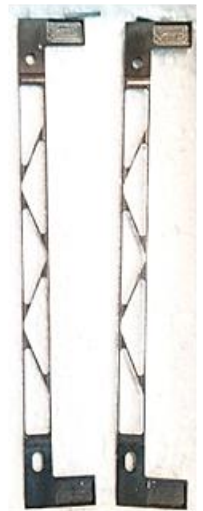

Final Al-CF part

Fig. 3. Al-CF Bridge fabrication process

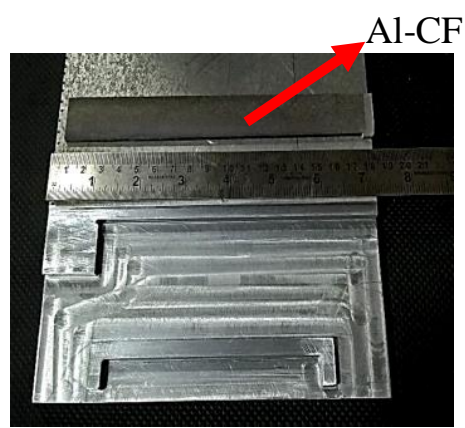

Initial

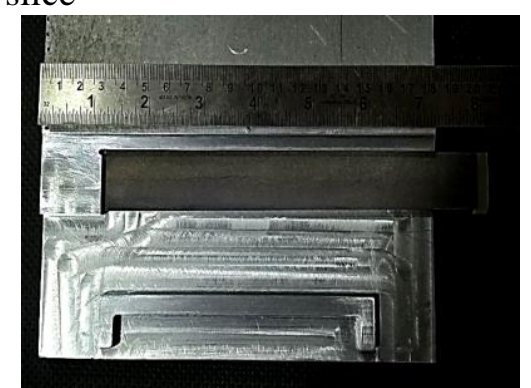

Stage 1

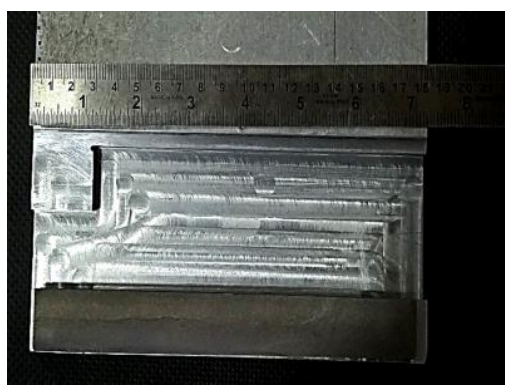

Stage 2

Fig. 4. Al-CF bridge fixture

Precise control over the accuracy of the fixture was important as the bridge structure was very fragile and even a slight deviation leads to the generation of bending moment resulting to the failure of the structure itself.

Macro-abrasive waterjet machine tool, Maxiem 1515 was used for slicing the part from the main Al-CF block. Waterjet pressure employed was 300MPa and garnet abrasive of $80 \#(185 \mathrm{~mm})$ was employed. The jet was traversed for cutting the desired geometry at a speed of $75 \mathrm{~mm} / \mathrm{min}$ with a standoff distance of $1.5 \mathrm{~mm}$. Orifice diameter of $0.35 \mathrm{~mm}$ and nozzle diameter of $0.76 \mathrm{~mm}$ were used for this cutting trials. Further, Jyoti K8mill was used for reducing the thickness and generating the profile on the sliced Al-CF. The machine tool has a maximum spindle speed of $15000 \mathrm{rpm}$, maximum feed rate of $10 \mathrm{~m} / \mathrm{min}$ with positional accuracy of $0.01 \mathrm{~mm}$. Solid carbide endmill tool of $1.5 \mathrm{~mm}$ diameter with spindle speed between $3000-5000 \mathrm{rpm}$ and feed rate of $50-100 \mathrm{~mm} / \mathrm{min}$ for machining the Al-CF stump. Further, Finecut WMC500II equipped with 
$22 \mathrm{~kW}$ pump and capable of pressurizing water up to a pressure of $4000 \mathrm{bar}$ with a maximum water flow rate of $2.31 / \mathrm{min}$ was used for cutting the complex structure in the bridge. It was observed that the approach followed was successful for the generation of Al-CF bridge structure.

In the second approach, the slices of Al-CF $(2.5 \mathrm{~mm})$ was cut from the main block by using bandsaw. A traverse rate of about $10 \mathrm{~mm} / \mathrm{min}$ was used for slicing while maintaining the cutting speed at $50 \mathrm{~m} / \mathrm{min}$. we maintaining the low traverse rate to maintain the accuracy of the geometry. Further, Jyoti K8mill was used for the generation of the bridge structure without employing the micro-abrasive waterjets for cutting the structure. The same process parameters were employed for the machining process as mentioned in the previous approach. Although, this approach was successful in the generation of Al-CF bridge structure, it was observed that 3 out of 7 samples failed after the whole structure was fabricated. This was considered to be due to the uneven surface and the thermal stresses generated on the sliced part. This approach is not considered to be a viable approach for high production.

\subsection{Al-CF Stump}

Three different approaches were identified for the fabrication of Al-CF stump structure For all the approaches followed, slicing from the main block was the first machining operation, which is followed by other machining processes for generating the required finished product. Figure 5 shows the dimensions and the geometry of the Al-CF stump structure. It can be seen that the part accuracy required is about $30 \mu \mathrm{m}$ and hence, the fabrication process needs to be precisely controlled.

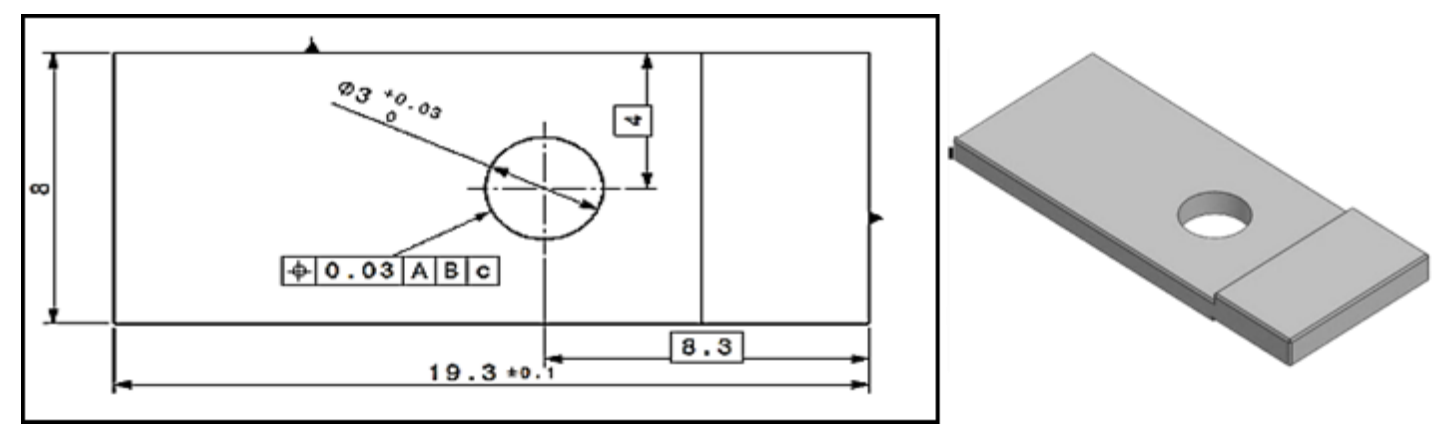

Fig. 5. Aluminum-Carbon fiber stump 


\subsubsection{Macro abrasive waterjet (AWJ) machining of Al-CF Stump}

Macro-AWJs were employed for both slicing and drilling operations. Waterjet pressure of $300 \mathrm{MPa}$ and garnet abrasives of $80 \#$ were used during the experiment. The same process parameters for slicing and drilling as mentioned in the previous section were used. A sacrificial layer was placed below the sliced part for supporting the part during the operation. It was observed that the slices broke during the profiling operation due to the backflow waterjet. Further, the fragile nature of the Al-CF structure made it prone to breakage even with less backflow waterjets. This approach was considered not viable for the production of Al-CF stump bridge structure.

\subsubsection{Wire Electric discharge machine sliced and milling of Al-CF stump}

In the second approach, the slices were cut using wire-electric discharge machining (WEDM) and the machining was done with milling process. Accutex Au-300iA CNC WEDM machine was used for slicing by employing brass wire diameter of $25 \mu \mathrm{m}$. However, the sliced part developed bends along the length due to the thermal stresses generated on the part during the WEDM machining process (Fig. 6).

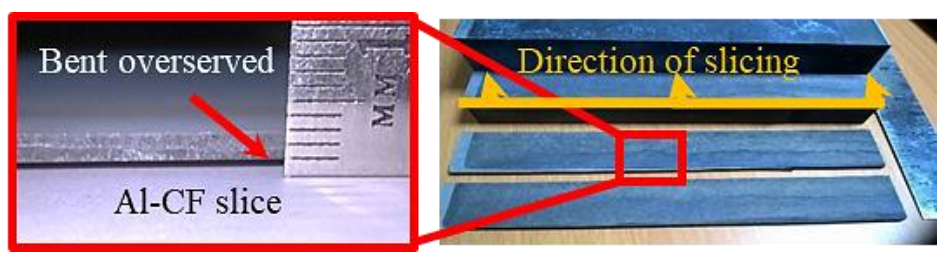

Fig. 6. Al-CF stumps fabrication process by WEDM and milling

Although the bending was observed along the total length of the Al-CF sliced part ( $200 \mathrm{~mm})$, there was less bending over the length required for fabricating the stump bridge $(19.3 \mathrm{~mm})$. Jyoti K8Mill was used for machining the structure using the same process parameters used for the fabrication of the Al-CF bridge strcuture. This approach was identified as one of the feasible processes for the fabrication of Al-CF stump bridge structure.

\subsubsection{Macro AWJ scling and milling of Al-CF stump}

In the third approach, macro-AWJs were used for slicing the Al-CF from the main block and milling for profiling and reducing the thickness (Fig. 7). There was no bending of the slices as there is negligible heat generated during the process. However, the final product 
developed certain burrs along the edges which can be avoided by optimizing the process parameters employed. This approach was identified as one of the feasible process for the fabrication of Al-CF bridge structure.

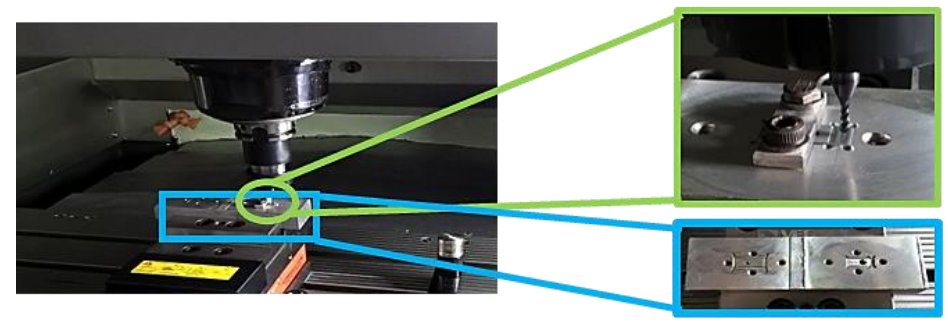

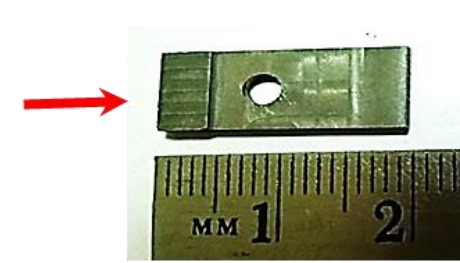

Finished part

Fig. 7. Al-CF stumps fabrication process by AWJ and milling

\subsection{Pipe connector 47 degrees}

For the fabrication of Al-CF pipe connector, only one approach was inentified as per the complexity of the structure and the material constriants. Slices of $6 \mathrm{~mm}$ were cut from the main Al-CF base structure using macro-AWJs by employing the same process parameters mentioned in the previous section. Further, the desired structure was generated by using WEDM (Fig. 8). EZEE CUT NXG capable of cutting with a resolution of $0.001 \mathrm{~mm}$ with wire diameter of 0.2 $0.25 \mathrm{~mm}$. A feed rate of $85 \mathrm{~mm} / \mathrm{min}$ was used for cutting the Al-CF pipe connector from the sliced block.
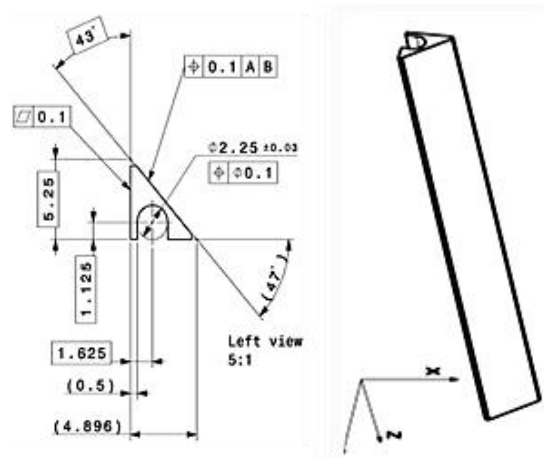

Part geometry for pipe connector

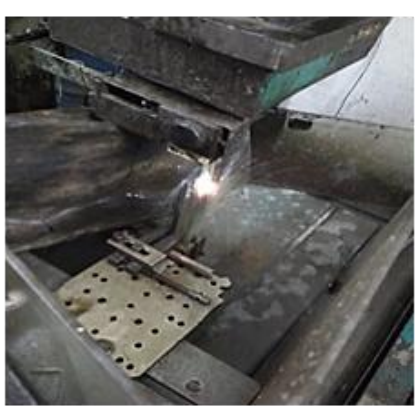

Machining process - wire EDM

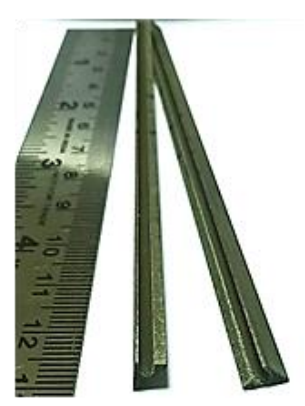

Machined part

Fig. 8. Al-CF pipe connector fabrication WEDM process

It was seen that the dimensions obtained conforms to the design. However, the whole process was time consuming and needs to be reduced for improving the productivity.

\section{Conclusion}

In this work, Al-CF pipe connector, bridge and stump bridge structures were fabricated by using various machining approaches. Following are the conclusions from this work 
- Multi-stage machining approach for the fabrication of Al-CF bridge was identified

- Two methods for the fabrication of Al-CF bridge were identified

- Al-CF pipe connector fabrication was possible by employing macro-AWJ slicing and WEDM

Although, the parts fabricated conforms to the desired dimensions, optimization of the whole process is required for an improved productivity.

\section{References}

[1] Behera, P. K., Singh, N. P., Devadula, S., and Alibordi, M., 2017, "Development of CMS Silicon Strip Detector Module Mechanics for Phase-II Upgrade," The 26th International Workshop on Vertex Detectors, Asturias, Spain, pp. 0-6.

[2] Abrate, S., and Walton, D. A., 1992, "Machining of Composite Materials. Part I: Traditional Methods,” Compos. Manuf., 3(2), pp. 75-83.

[3] Srinivasu, D. S., and Axinte, D. A., 2014, "Mask-Less Pocket Milling of Composites by Abrasive Waterjets: An Experimental Investigation,” J. Manuf. Sci. Eng., 136(4), p. 041005.

[4] Li, H., and Wang, J., 2015, “An Experimental Study of Abrasive Waterjet Machining of Ti-6Al4V,” Int. J. Adv. Manuf. Technol., 81(1-4), pp. 361-369.

[5] Karakurt, I., Aydin, G., and Aydiner, K., 2012, "An Experimental Study on the Depth of Cut of Granite in Abrasive Waterjet Cutting," Mater. Manuf. Process., 27(5), pp. 538-544. 